$02,05,13$

\title{
Экспериментальное исследование роли триплетного спаривания в эффекте сверхпроводящего спинового клапана
}

\author{
(c) П.В. Лексин ${ }^{1,4}$, А.А. Камашев ${ }^{1}$, Н.Н. Гарифььянов ${ }^{1}$, А.А. Валидов ${ }^{1}$, Я.В. Фоминов ${ }^{2,3}$, \\ J. Schumann ${ }^{4}$, В.Е. Катаев ${ }^{4}$, В. Вüchner ${ }^{4,5}$, И.А. Гарифруллин ${ }^{\mp, 1}$ \\ ${ }^{1}$ Казанский фризико-технический институт им. Е.К. Завойского КазНЦ РАН, \\ Казань, Россия \\ ${ }^{2}$ Институт теоретической фризики им. Л.Д. Ландау РАН, \\ Черноголовка, Россия \\ ${ }^{3}$ Московский фризико-технический институт (Государственный университет), \\ Долгопрудный, Россия \\ ${ }^{4}$ Leibniz Institute for Solid State and Materials Research IFW Dresden, \\ Dresden, Germany \\ ${ }^{5}$ Institut für Festkörperphysik, Technische Universität Dresden, \\ Dresden, Germany \\ ๑ E-mail: ilgiz0garifullin@gmail.com
}

\begin{abstract}
Установлена важная роль морфологии сверхпроводящего слоя в работе сверхпроводящего спинового клапана. Для образцов $\mathrm{CoO}_{x} / \mathrm{Py} 1 / \mathrm{Cu} / \mathrm{Py} 2 / \mathrm{Cu} / \mathrm{Pb}$ (где $\mathrm{Py}=\mathrm{Ni}_{0.81} \mathrm{Fe}_{0.19}$ ) с гладким сверхпроводящим слоем экспериментально изучено триплетное спаривание, индуцированное эффектом близости сверхпроводник/ферромагнетик. Оптимизация параметров этой структуры позволила продемонстрировать полное переключение между нормальным и сверхпроводящим состояниями при изменении взаимной ориентации намагниченностей ферромагнитных слоев от антипараллельной к ортогональной. Для образца с толщиной пермаллоя, при которой величина эффекта спинового клапана обращается в нуль, наблюдался „чистый“ триплетный вклад. Впервые проведено прямое сравнение экспериментальных данных с теоретическим расчетом температуры перехода в сверхпроводящее состояние.
\end{abstract}

Работа частично поддерживалась грантами DFG (грант LE 3270/1-1), РФФИ (грант 14-02-00350-а), Программами РАН, Министерством образования и науки Российской Федерации и программой „5top $100 “$.

\section{1. Введение}

Эффект сверхпроводящего спинового клапана состоит в различной степени подавления сверхпроводимости в тонкопленочных мультислоях F1/F2/S или F1/S/F2 для параллельной (Р) и антипараллельной (АР) взаимной ориентации намагниченностей ферромагнитных слое F1 и F2 [1]. Функционирование сверхпроводящего спинового клапана, основанного на эффекте близости сверхпроводник/ферромагнетик (S/F), дает ключ к пониманию многих фундаментальных аспектов взаимодействия сверхпроводимости и магнетизма. Спиновый клапан имеет хорошие перспективы для его использования в качестве пассивного элемента сверхпроводниковой спинтроники. Эта конструкция должна функционировать путем изменения направления небольшого внешнего магнитного поля. Для подтверждения наличия этого эффекта для систем S/F c хорошим контактом между металлическими ферромагнитными и сверхпроводящими слоями, сделанными из обычных металлов и стандартных ферромагнетиков, было выполнено большое число экспериментальных работ (см., например, недавние обзоры [2-4] и ссылки в них). Несмотря на различные величины эффекта спинового клапана $\Delta T_{c}=T_{c}^{\mathrm{AP}}-T_{c}^{\mathrm{P}}$ $\left(\Delta T_{c}=10 \mathrm{mK}\right.$ в [5], $\Delta T_{c}=20 \mathrm{mK}$ в [6] и $\Delta T_{c}=120 \mathrm{mK}$ в [7]), полное переключение между сверхпроводящим и нормальным состояниями было реализовано только в двух случаях $[8,9]$. Это связано с тем, что обычно $\Delta T_{c}$ оказывалось меньше ширины сверхпроводящего перехода $\delta T_{c}$.

Недавно Сингх и др. [10] доложили о наблюдении колоссального триплетного эффекта спинового клапана для структуры $\mathrm{S} / \mathrm{F} 1 / \mathrm{N} / \mathrm{F} 2$, сделанной из аморфного $\mathrm{MoGe}, \mathrm{Ni}, \mathrm{Cu}$ и $\mathrm{CrO}_{2}$ в качестве слоев $\mathrm{S}, \mathrm{F} 1, \mathrm{~N}$ и $\mathrm{F} 2$ соответственно. При изменении взаимной ориентации ферромагнитных слоев в этой структуре температура перехода в сверхпроводящее состояние $T_{c}$ изменялось примерно на $1 \mathrm{~K}$, что намного превышает полученные ранее значения. Было показано, что оптимальное рабочее поле для этой конструкции составляет $20 \mathrm{kOe}$. Практически в то же время Гу идр. [11,12] сообщили о наблюдении $\Delta T_{c} \sim 400 \mathrm{mK}$ для трехслойной системы $\mathrm{Ho} / \mathrm{Nb} / \mathrm{Hl}$. В этом случае параллельная конфигурация намагниченностей достигается в поле порядка $10 \mathrm{kOe}$. Большие рабочие поля этих конструкций являются большим недостатком для сверхпроводниковой спинтроники. Помимо этого, природа больших значений $\Delta T_{c}$ в спиновом клапане на основе полуметаллического ферромагнетика [10] еще не имеет окончательного теоретического объяснения. Первая попытка объяснить колоссальный триплетный эффект спинового клапана, содержащего полуметалл в качестве F-слоя, была предпринята в работе Миронова и Буздина [13]. Это говорит о том, что исследование простейших структур сверхпроводящего 
Таблица 1. Параметры I и II серии образцов

\begin{tabular}{c|c}
\hline Серия образца & Параметры структуры \\
\hline Ia & $\operatorname{Py}(5 \mathrm{~nm}) / \mathrm{Pb}(x), x=60,70,75,90,105,150,240 \mathrm{~nm}$ \\
Ib & $\operatorname{Py}(5 \mathrm{~nm}) \mathrm{Cu}(2 \mathrm{~nm}) \operatorname{Pb}(x), x=70,90,200 \mathrm{~nm}$ \\
Ic & $\operatorname{CoO} \operatorname{Py} 1(3 \mathrm{~nm}) \mathrm{Cu}(4 \mathrm{~nm}) \operatorname{Py} 2(1 \mathrm{~nm}) \mathrm{Cu}(2 \mathrm{~nm}) \operatorname{Pb}(70 \mathrm{~nm})$ \\
IIa & $\operatorname{Py}(5 \mathrm{~nm}) \operatorname{Pb}(x), x=70,95,120 \mathrm{~nm}$ \\
IIb & $\operatorname{Py}(5 \mathrm{~nm}) \mathrm{Cu}(2 \mathrm{~nm}) \operatorname{Pb}(x), x=47,70,80,94,110,125,155 \mathrm{~nm}$ \\
IIc & $\operatorname{CoO}_{x} \operatorname{Py} 1(3 \mathrm{~nm}) \mathrm{Cu}(4 \mathrm{~nm}) \operatorname{Py} 2(1 \mathrm{~nm}) \mathrm{Cu}(2 \mathrm{~nm}) \operatorname{Pb}(70 \mathrm{~nm})$
\end{tabular}

спинового клапана, позволяющих реализовать желаемую ситуацию, остается актуальной. В таких структурах в качестве ферромагнитных слоев используются классические ферромагнитные материалы (Fe, $\mathrm{Co}, \mathrm{Ni}$ ) и их сплавы, а также существует хороший электрический контакт между всеми слоями.

Недавние теоретические работы (см., например, недавние обзоры [2-4,14-17]) предсказывают генерацию дальнодействующей триплетной компоненты (ДТК) сверхпроводящего конденсата в S/F-структурах. Как следует из теории Фоминова и др. [18], наличие минимума $T_{c}$ вблизи ортогональной конфигурации намагниченностей однозначно свидетельствует о генерации ДТК. Мы получили экспериментальное подтверждение существования ДТК для структуры сверхпроводящего спинового клапана $\mathrm{CoO}_{x} / \mathrm{Fe} 1 / \mathrm{Cu} / \mathrm{Fe} 2 / \mathrm{Pb}$ [19]. Наблюдаемая угловая зависимость $T_{c}$ была вызвана комбинацией синглетной и триплетной компонент сверхпроводящего конденсата. Наличие триплетного вклада в величину эффекта сверхпроводящего спинового клапана было также показано в работах [5-7,20,21].

Возможность наблюдения „изолированного“ триплетного вклада в эффект спинового клапана является важным вопросом для фундаментальных и прикладных исследований в области спинтроники. На первый взгляд это кажется невозможным, потому что ДТК возникают из синглетных компонент и существовать без них не могут. Однако, в данной работе мы экспериментально наблюдали „изолированный“ триплетный эффект, который был получен за счет осцилляционного поведения $\Delta T_{c}\left(d_{\mathrm{F} 2}\right)$, при котором наблюдается подавление обычного эффекта спинового клапана при соответствующем выборе толщины слоя $\mathrm{F} 2 d_{\mathrm{F} 2}$.

\section{2. Образцы}

2.1. Выбор материала ферромагнитного с лоя. В 2013 году нами были проведены исследования зависимости величины эффекта сверхпроводящего спинового клапана от толщин слоев F1 и F2 в структурах $\mathrm{CoO}_{x} / \mathrm{Fe} 1 / \mathrm{Cu} / \mathrm{Fe} 2 / \mathrm{Cu} / \mathrm{Pb}[22,23]$. Из анализа полученных экспериментальных данных было установлено, что в соответствии с теорией [18] максимальная величина эффекта сверхпроводящего спинового клапана $\Delta T_{c}$ должна наблюдаться при толщинах слоев F1 и F2 порядка половины глубины проникновения куперовских пар в ферромагнетик $\xi_{h}$, который для железа составляет величину порядка $0.8 \mathrm{~nm}$. В эксперименте на нашем оборудовании нам не удается напылить образцы с толщиной слоя $\mathrm{Fe} \sim 0.5 \mathrm{~nm}$ и меньше. Это связано с тем, что слой железа при таких толщинах становится несплошным, вследствие начального островкового роста, и его воздействие на сверхпроводимость становится неоднозначным. Универсальной координатой толщин в зависимости $\Delta T_{c}\left(d_{\mathrm{Fe} 1,2}\right)$ является отношение $d_{\mathrm{F}} / \xi_{h}$, где $\xi_{h}-$ глубина проникновения куперовской пары в ферромагнетик. Это говорит о том, что для того, чтобы выйти в область достижимых нами толщин F-слоя необходимо увеличивать $\xi_{h}=\sqrt{\hbar D_{\mathrm{F}} / h}$, где $D_{\mathrm{F}}-$ коэффициент диффузии электронов в F-слое, $h$ - обменное поле в ферромагнетике. Следовательно, для того, чтобы наблюдать теоретически предсказанный максимум $\Delta T_{c}$ необходимо выбрать ферромагнетик с меньшим значением обменного поля $h$, чем у железа. В качестве первого и наиболее простого шага в этом направлении мы решили использовать пермаллой $\left(\right.$ Ру $\left.=\mathrm{Ni}_{0.81} \mathrm{Fe}_{0.19}\right)$, хотя, априори, и не очевидно, что величина $h$ в пермаллое меньше, чем в железе. Тем не менее, наши исследования зависимости $T_{c}$ толщины слоя пермаллоя показали, что величина обменного поля в пермаллое приблизительно в 2 раза меньше чем, в железе.

2.2. Морфология сверхпроводящего слоя по данным просвечиваюей электронной м икр оскопи и. Для начала мы детально исследовали влияние условий приготовления образцов и их дизайна на морфологию. Для этого был использован просвечивающий электронный микроскоп FEI TEM/STEM Technai F30 при ускоряющем напряжении $30 \mathrm{kV}$. Прозрачные для электронов поперечные срезы были приготовлены с помощью сфокусированного ионного пучка в установке Zeiss 1540XB. Рентгеновская дисперсионная спектроскопия подтвердила присутствие тонкого слоя пермаллоя (см. [24]). Границы раздела между отдельными слоями отчетливо видны на рис. 1. Просвечивающая электронная микроскопия показала, что в структуре $\mathrm{MgO} / \mathrm{Py} / \mathrm{Pb}$, приготовленной при температуре подложки $300 \mathrm{~K}$, слой свинца растет в виде перекрывающихся островков с размерами 0.2-1 $\mu \mathrm{m}$ (см. рис. $1, b, c)$. Что же касается структуры $\mathrm{MgO} / \mathrm{Py} / \mathrm{Cu} / \mathrm{Pb}$, напыленной при температуре подложки $150 \mathrm{~K}$, то изображение, полученное с помощью просвечивающей электронной микроскопии, свидетельствует о гладкой поверхности слоя свинца и границ раздела $\mathrm{Py} / \mathrm{Cu} / \mathrm{Pb}$ (см. рис. $1, e, f)$. Толщина 


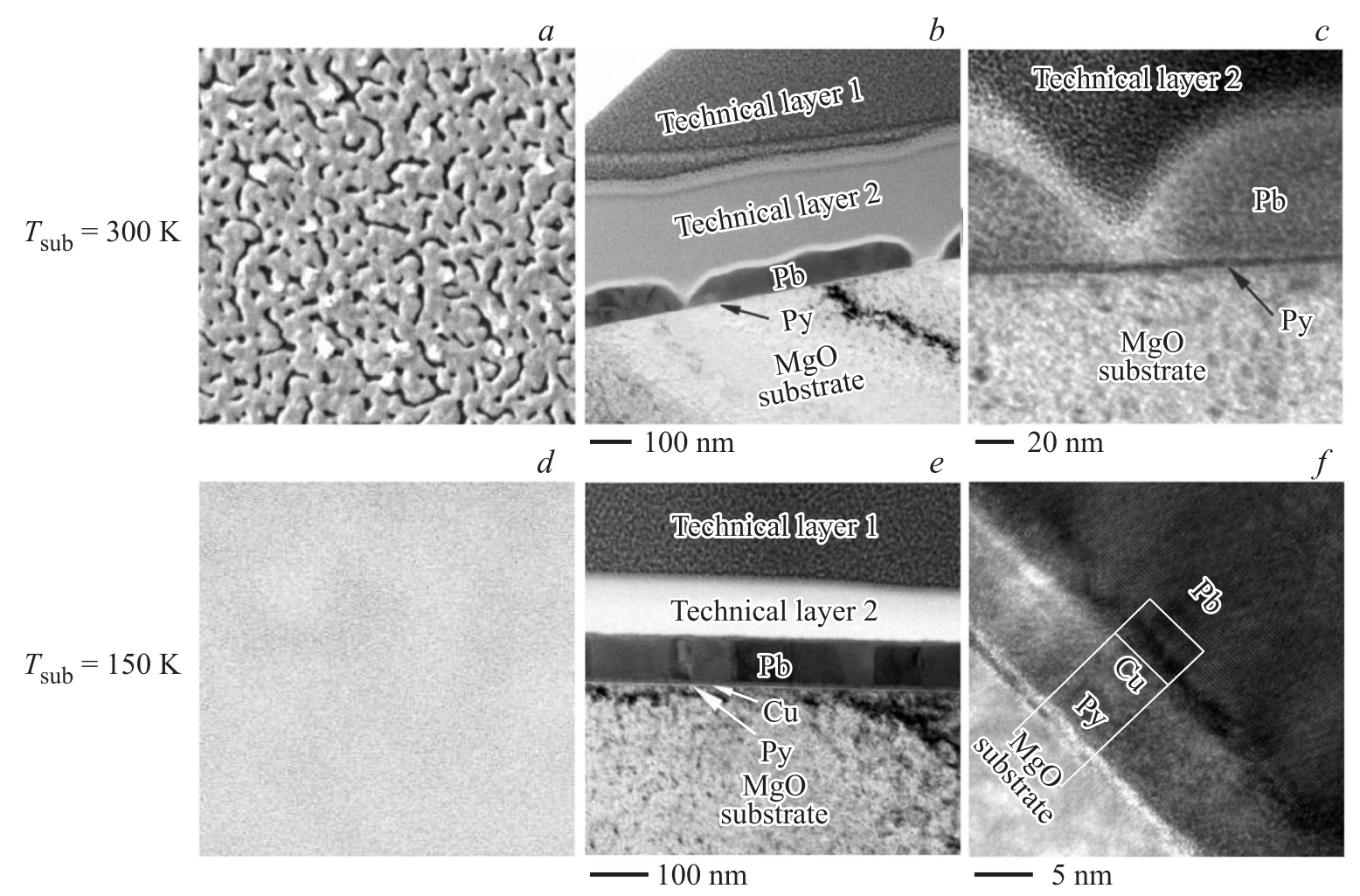

Рис. 1. Микрофотографии поверхности образцов, приготовленных при температурах подложки 300 и $150 \mathrm{~K}$ соответственно. Электронно-микроскопические изображения тонкого поперечного среза образца $\mathrm{Py}(5) / \mathrm{Pb}(70)$, приготовленного при температуре подложки $300 \mathrm{~K}$, представлены на частях $(a),(b)$ и $(c)$; на $(d),(e)$ и $(f)$ представлены изображения для образца $\mathrm{Py}(5) / \mathrm{Cu}(2) / \mathrm{Pb}(70)$, приготовленного при температуре подложки $150 \mathrm{~K}$.

a
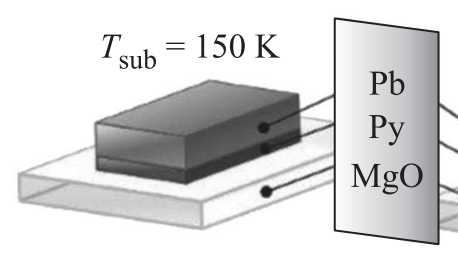

$T_{\text {sub }}=300 \mathrm{~K}$
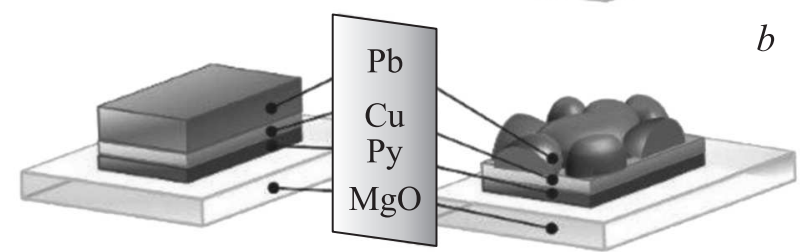

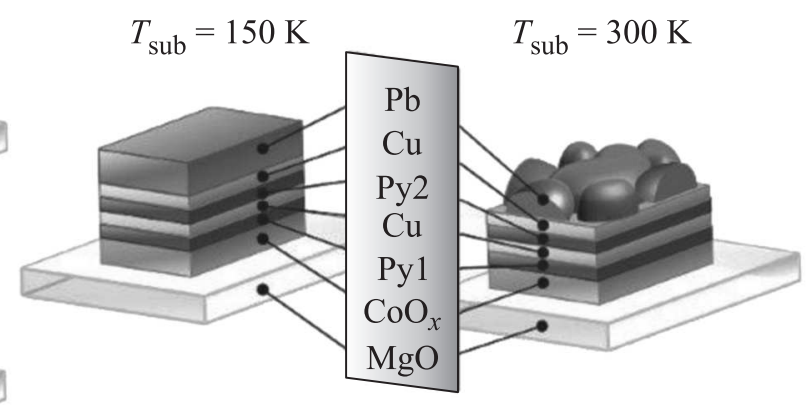

Рис. 2. Схематическое изображение образцов: двухслойные структуры $(a)$, трехслойные структуры $(b)$ и структуры сверхпроводящего спинового клапана $(c)$ с шероховатыми (правая сторона) и гладкими (левая сторона) $S$-слоями.

сверхпроводящего слоя была оценена, как $d_{\mathrm{Pb}}=70 \mathrm{~nm}$. Слабый контраст между изображениями слоев $\mathrm{Cu}$ и Ру не позволил оценить их толщину по отдельности. Общая толщина слоев $\mathrm{Py} / \mathrm{Cu}$ составляет $8.6 \pm 0.1 \mathrm{~nm}$, что находится в согласии с номинальными толщинами слоев Ру и $\mathrm{Cu}, d_{\mathrm{Pb}}=5 \mathrm{~nm}$ и $d_{\mathrm{Cu}}=2 \mathrm{~nm}$.

2.3. Описание образцов. Список приготовленных образцов для исследований представлен в табл. 1. Согласно данным электронно-микроскопических исследований морфология этих образцов может быть схе- матически представлена так, как показано на рис. 2. Ранее в [22,23] нами было показано, что деградация сверхпроводящих свойств образцов, содержащих границу раздела $\mathrm{Pb} / \mathrm{Fe}$, может быть преодолена путем введения тонкого слоя меди между S- и F-слоями. Вместе с тем из сравнения рис. $2, a$ и $2, b$ видно, что этот слой не оказывает заметного влияния на морфологию сверхпроводящего слоя. Главным фактором оказывается температура подложки. На рис. 2, $c$ показано устройство образца спинового клапана. Здесь $\mathrm{MgO}$ - это 


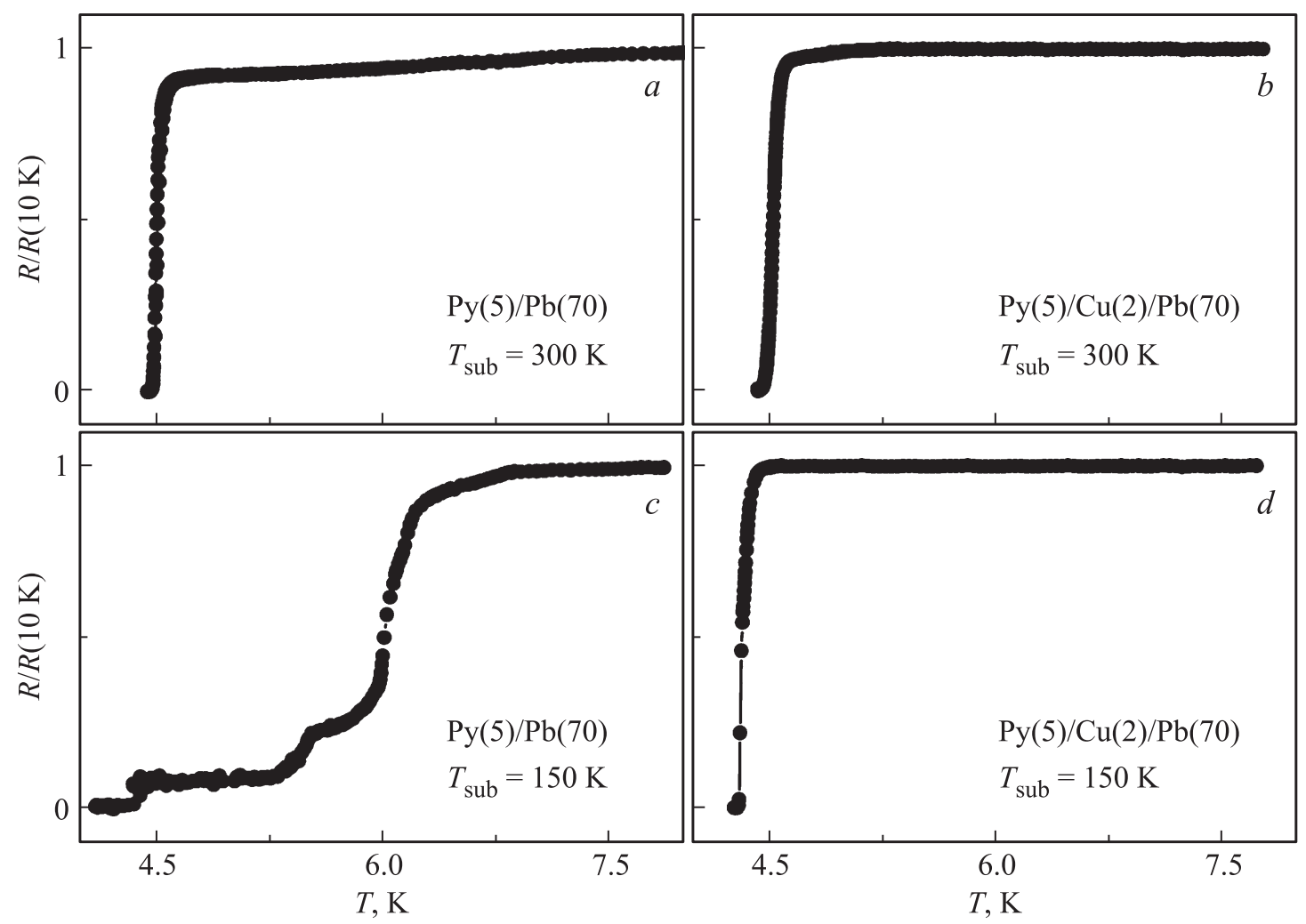

Рис. 3. Характеризация сверхпроводящих свойств образцов $\mathrm{Py}(5) / \mathrm{Pb}(70)$ и $\mathrm{Py}(5) / \mathrm{Cu}(2) / \mathrm{Pb}(70)$. (a), (b) - сверхпроводящие переходы для образцов с шероховатым слоем $\mathrm{Pb}\left(T_{\mathrm{sub}}=300 \mathrm{~K}\right) ;(c),(d)-$ сверхпроводящие переходы для образцов с гладким слоем $\mathrm{Pb}\left(T_{\text {sub }}=150 \mathrm{~K}\right)$.

монокристаллическая подложка. $\mathrm{CoO}_{x}$ необходим для закрепления намагниченности слоя пермаллоя Ру1 при охлаждении образца в магнитном поле. При этом намагниченность слоя Ру2, отделенного от Ру1 слоем меди, оказывается свободной и может поворачиваться слабым магнитным полем. И наконец, слой свинца играет роль сверхпроводника.

Образцы были приготовлены классическим методом электронно-лучевого испарения в сверхвысоком вакууме на установке МВЕ. Были изучены свойства образцов, полученных в двух различных режимах. Серии образцов Ia, Ib и Ic были получены при напылении на подложку, находящуюся при комнатной температуре, a серии образцов IIa, IIb и IIc - при температуре подложки $150 \mathrm{~K}$. Для напыления слоев использовались следующие скорости напыления: $0.5 \AA / \mathrm{s}$ для слоев Ру и $\mathrm{Cu} ; 2 \AA / \mathrm{s}$ и $12 \AA / \mathrm{s}$ для шероховатых и гладких слоев свинца, соответственно.

\section{3. Экспериментальные результаты}

3.1. Влияние морфологии сверхпроводящего слоя на свойства образцов. Температура сверхпроводящего перехода образцов измерялась по изменению электросопротивления 4-х контактным методом. Было установлено, что отношение удельных значений электросопротивлений $R R R$, исследованных нами образцов, лежит в интервале 10-17. Такие значения $R R R$ свидетельствуют о высокой чистоте слоя $\mathrm{Pb}$ в наших образцах. На рис. 3 представлены характерные сверхпроводящие переходы для образцов с шероховатым и гладким S-слоем для структур $\mathrm{Py} / \mathrm{Pb}$ и $\mathrm{Py} / \mathrm{Cu} / \mathrm{Pb}$. Сравнение сверхпроводящих переходов образцов $\mathrm{Py} / \mathrm{Pb}$ и $\mathrm{Py} / \mathrm{Cu} / \mathrm{Pb}$, приготовленных при температуре подложки $300 \mathrm{~K}$ (см. рис. $3, a$ и рис. $3, b$ ), показывает, что дополнительный слой меди не оказывает влияния на степень подавления $T_{c}$ в этих структурах. Для обеих структур сверхпроводящие переходы оказываются узкими с одинаковым значением $T_{c}$. Противоположные результаты были получены для образцов Ру/Pb, приготовленных при температуре подложки $150 \mathrm{~K}$ (см. рис. $3, c$ ). Сверхпроводящие переходы демонстрируют несколько ступенек, свидетельствующих о фазовом расслоении на границе раздела $\mathrm{Py} / \mathrm{Pb}$. Введение дополнительного слоя меди между слоями $\mathrm{Py}$ и $\mathrm{Pb}$ коренным образом изменяет качество сверхпроводящего перехода (см. рис. $3, d$ ), делая его более совершенным для структур.

Влияние ферромагнитного порядка на сверхпроводящие свойства структуры F1/F2/S зависит от нескольких параметров сверхпроводящего слоя. Один из самых важных параметров - толщина сверхпроводящего слоя $\left(d_{S}\right)$. Чем тоньше сверхпроводящий слой, тем сильнее подавляется $T_{c}$, и, следовательно, можно ожидать большего значения величины эффекта сверхпро- 


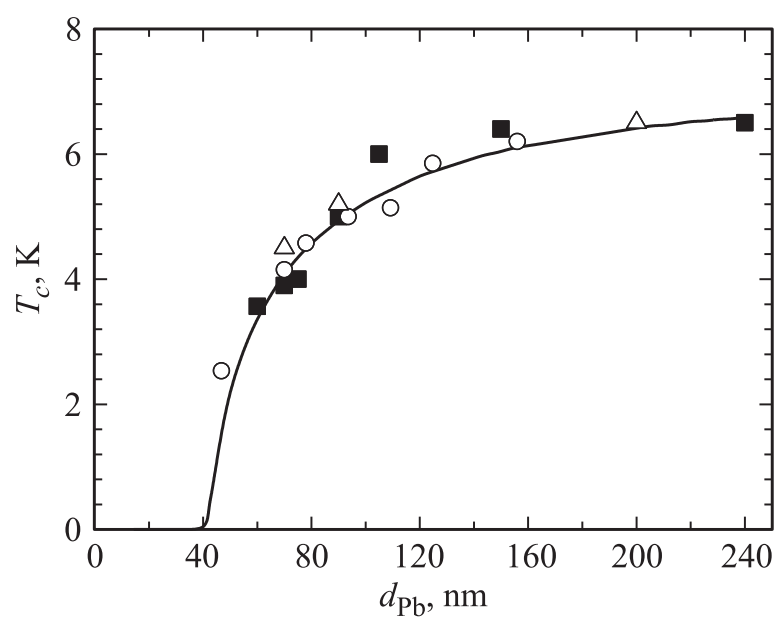

Рис. 4. Зависимость $T_{c}$ от толщины сверхпроводящего слоя $\mathrm{Pb}$ $d_{\mathrm{Pb}}$ для структур $\mathrm{Py} / \mathrm{Pb}$ и $\mathrm{Py} / \mathrm{Cu} / \mathrm{Pb}$ с шероховатым слоем свинца (квадратики и открытые треугольники), и для структур $\mathrm{Py} / \mathrm{Cu} / \mathrm{Pb}$ с гладким слоем свинца (светлые кружки). Сплошная линия - теоретическая кривая, рассчитанная согласно теории [26].

водящего спинового клапана. Как правило, оптимальную толщину сверхпроводящего слоя определяют из зависимости $T_{c}\left(d_{S}\right)$. В принципе, такую кривую можно рассчитать и теоретически (см. [25]). Для того чтобы экспериментально установить влияние морфологии сверхпроводящего слоя, были проведены измерения зависимости $T_{c}$ от толщины слоя $\mathrm{Pb} d_{\mathrm{Pb}}$ для структур $\mathrm{Py} / \mathrm{Pb}$ и $\mathrm{Py} / \mathrm{Cu} / \mathrm{Pb}$ с шероховатым и гладким сверхпроводящим слоем при фиксированной толщине $d_{\mathrm{Py}}=5 \mathrm{~nm}$, которая намного превышает глубину проникновения $\xi_{h}$ куперовских пар в ферромагнитный пермаллой. Зависимость, представленная на рис. 4, позволила определить оптимальную толщину S-слоя. Из рис. 4 видно, что при больших толщинах свинца $T_{c}$ медленно уменьшается с уменьшением $d_{\mathrm{Pb}}$. Ниже $d_{\mathrm{Pb}} \sim 120 \mathrm{~nm}$ значение $T_{c}$ начинает резко уменьшаться. При $d_{\mathrm{Pb}}<40 \mathrm{~nm} T_{c}$ становится меньше $1.5 \mathrm{~K}$. При $d_{\mathrm{Pb}}<70 \mathrm{~nm}$ ширина сверхпроводящего перехода оказывается очень большой, порядка $0.4 \mathrm{~K}$. Имея в виду, что влияние магнитного слоя структуры тем сильнее, чем ближе толщина сверхпроводящего слоя к длине когерентности сверхпроводника (для наших образцов $\left.\xi_{S}=41 \mathrm{~nm}\right) d_{\mathrm{Pb}}=70 \mathrm{~nm}$ была выбрана как оптимальная толщина.

Как видно из рис. 4, образцы показывают схожее поведение $T_{c}\left(d_{\mathrm{Pb}}\right)$, подтверждая, что дополнительный слой меди не оказывает влияния на эффект близости $\mathrm{S} / \mathrm{F}$. Представленные на рис. 4 зависимости демонстрируют также отсутствие влияния морфологии сверхпроводящего слоя образцов на характер подавления $T_{c}$. Сплошной линией на рис. 4 показана теоретическая кривая $T_{c}\left(d_{\mathrm{Pb}}\right)$, рассчитанная в соответствии с теорией Фоминова и др. (см. [25]). Был проведен сравнительный анализ экспериментальных данных с теоретическими расчетами Фоминова и др., используя следующие параметры: длины когерентности для S- и F-слоев $\xi_{S}=41 \mathrm{~nm}, \xi_{\mathrm{F}}=13 \mathrm{~nm}$; величина обменного поля в ферромагнетике $h=0.3 \mathrm{eV}$; длина свободного пробега электронов в сверхпроводнике $l_{S}=30 \mathrm{~nm}$, в ферромагнетике $l_{\mathrm{F}}=3 \mathrm{~nm}$; параметры прозрачности для границы $\mathrm{S} / \mathrm{F} \gamma=0.74$ и $\gamma_{b}=2.2$. Анализ показал удовлетворительное согласие теории и экспериментальных данных (см. рис. 4). На основе зависимости $T_{c}\left(d_{\mathrm{Py}}\right)$ при фиксированном значении $d_{\mathrm{Pb}}$ была выполнена оценка величины $\xi_{h}$ : полученное значение составляло $1.1 \mathrm{~nm}$.

Для структуры сверхпроводящего спинового клапана $\mathrm{CoO}_{x} / \mathrm{Py}(3) / \mathrm{Cu}(4) / \mathrm{Py}(1) / \mathrm{Cu}(2) / \mathrm{Pb}(70)$ с шероховатым и гладким сверхпроводящим слоем характеризация при помощи SQUID-магнитометра не выявила различий в магнитных свойствах для этих структур. При этом

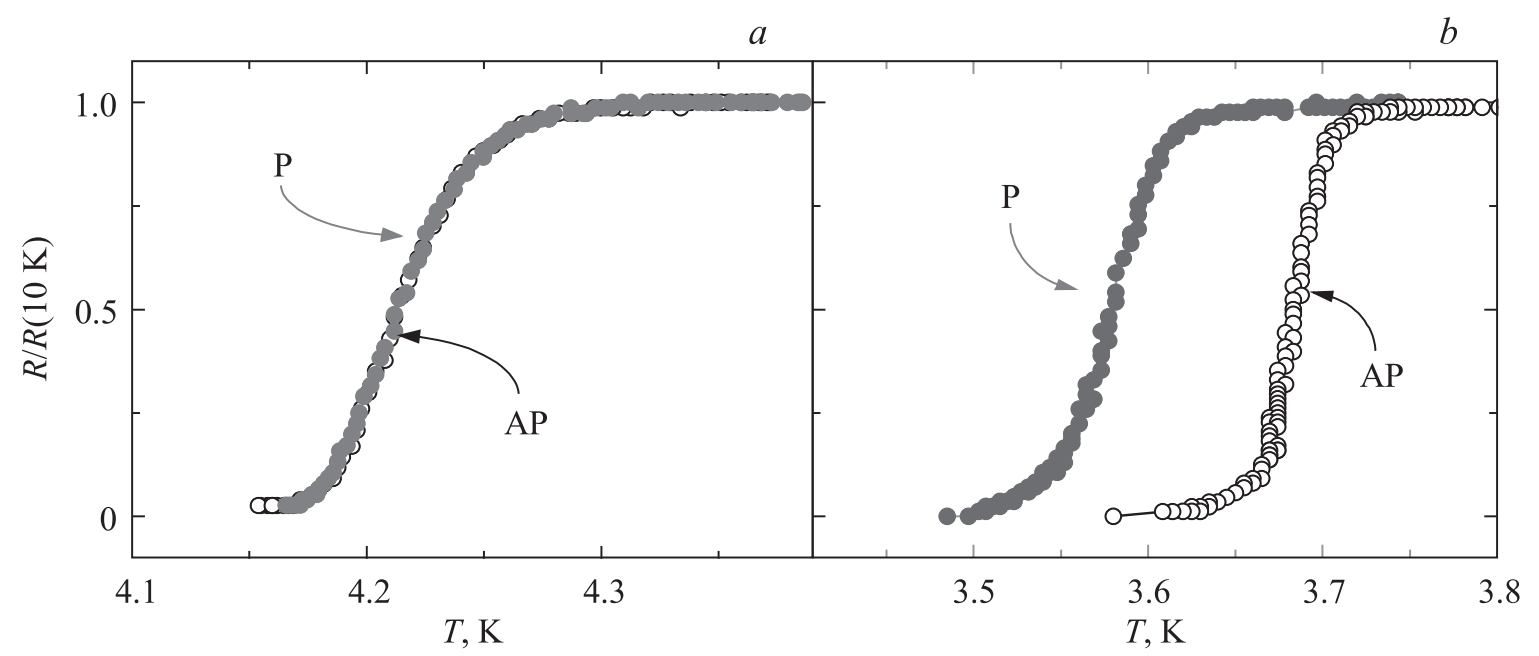

Рис. 5. Сверхпроводящие переходы, измеренные при AP (открытые кружки) и Р (закрытые кружки) ориентациях намагниченностей ферромагнитных слоев, для образцов $\mathrm{CoO}_{x} / \mathrm{Py}(3) / \mathrm{Cu}(4) / \mathrm{Py}(1) / \mathrm{Cu}(2) / \mathrm{Pb}(70)$ с шероховатым (a) и гладким (b) сверхпроводящим слоем. 
Таблица 2. Параметры 3 серии образцов

\begin{tabular}{c|c|c|c|l|c|c|c}
\hline \multirow{2}{*}{ Образец } & \multicolumn{7}{|c}{ Толщина слоев, $\mathrm{nm}$} \\
\cline { 2 - 8 } & $\mathrm{CoO}_{x}$ & $\mathrm{Py} 1$ & $\mathrm{Cu}$ & $\mathrm{Py} 2$ & $\mathrm{Cu}$ & $\mathrm{Pb}$ & $\mathrm{Si}_{3} \mathrm{~N}_{4}$ \\
\hline $3 \mathrm{a}$ & 3 & 3 & 4 & 0.6 & 2 & 70 & 85 \\
$3 \mathrm{~b}$ & 3 & 3 & 4 & 1 & 2 & 70 & 85 \\
$3 \mathrm{c}$ & 3 & 3 & 4 & 1.7 & 2 & 70 & 85 \\
$3 \mathrm{~d}$ & 3 & 3 & 4 & 3 & 2 & 70 & 85
\end{tabular}

для структур с шероховатым сверхпроводящим слоем при переключении между AP и $\mathrm{P}$ ориентацией намагниченностей ферромагнитных слоев, величина эффекта сверхпроводящего спинового клапана $\Delta T_{c}$ оказалась меньше $10 \mathrm{mK}$ (см. рис. 5,a). В тоже время для структуры сверхпроводящего спинового клапана с гладким сверхпроводящим слоем, величина эффекта $\Delta T_{c}$ достигает $100 \mathrm{mK}$ и более (см. рис. $5, b)$.

3.2. Эффект сверхпроводящего спинового к лап ана. В этой части статьи изложены результаты исследования образцов сверхпроводящего спинового клапана $\mathrm{CoO}_{x} / \mathrm{Py} 1 / \mathrm{Cu} / \mathrm{Py} 2 / \mathrm{Cu} / \mathrm{Pb}$, которые были приготовлены при температуре подложки $150 \mathrm{~K}$, с варьируемыми толщинами слоя $d_{\text {Ру2 }}$. Основные параметры образцов были определены в вышеизложенном материале данной статьи. В табл. 2 приведены параметры третьей серии образцов.

На первом этапе, были исследованы магнитные свойства третьей серии образцов с помощью 7T VSM SQUID магнитометра (7 Tesla Vibrating Sample Magnetometer SQUID). Были проведены измерения зависимости намагниченности $M$ от магнитного поля $H$ для определения интервала магнитных полей, в которых достигаются АР и $\mathrm{P}$ ориентации намагниченностей слоев Ру1 и Ру2. На рис. 6, a приведена петля магнитного гистерезиса для образца $3 \mathrm{~b} \quad \mathrm{CoO}_{x} / \mathrm{Py}(3) / \mathrm{Cu}(4) / \mathrm{Py}(1) / \mathrm{Cu}(2) / \mathrm{Pb}(70)$. Поле прикладывалось вдоль оси легкого намагничивания, находящегося в плоскости образца вдоль длинной оси. В начале исследования магнитных свойств образцы охлаждались от комнатной температуры до температуры $10 \mathrm{~K}$ в магнитном поле $+4 \mathrm{kOe}$. Измерение намагниченности осуществлялось при температуре $10 \mathrm{~K}$. При этом магнитное поле изменялось от +4 до $-6 \mathrm{kOe}$ и обратно до значения $+4 \mathrm{kOe}$. Оба значения магнитного поля +4 и $-6 \mathrm{kOe}$ соответствуют параллельной ориентации намагниченностей слоев Ру1 и Ру2. В первом случае вдоль направления поля, в котором производилось охлаждение образца, во втором - в противоположной ориентации. Для всех образцов при уменьшении поля от +4 до $+0.2 \mathrm{kOe}$ намагниченность постоянна. Это соответствует тому, что намагниченности ферромагнитных пленок параллельны и находятся в насыщении. При дальнейшем уменьшении магнитного поля намагниченность слоя Ру2 начинает уменьшаться и выходит на плато при $H=-0.1 \mathrm{kOe}$. При этом, намагниченность слоя Ру1 остается зафиксированной в исходном направлении до приложения магнитного поля величиной $-2 \mathrm{kOe}$. Именно до этой величины магнитного поля слой Ру1 удерживается. Это ведет к тому, что в диапазоне магнитных полей от -0.1 до $-2 \mathrm{kOe}$ достигается антипараллельная взаимная ориентация намагниченностей слоев Py1 и Ру2. При дальнейшем изменении поля от -2 до $-2.5 \mathrm{kOe}$ намагниченность слоя Ру1 тоже начинает изменять свое значение, и в диапазоне магнитных полей от -2.5 до $-6 \mathrm{kOe}$ реализуется параллельная ориентация намагниченностей слоев пермаллоя в направлении противоположном начальной параллельной ориентации. На качественном уровне такой вид магнитных петель гистерезиса характерен для всех образцов из третьей серии.

Для изучения намагниченности промежуточного слоя пермаллоя Ру2 были записаны также малые петли гистерезиса. На рис. $6, b$ приведена малая петля гистерезиса. Измерения проводились следующим образом. После
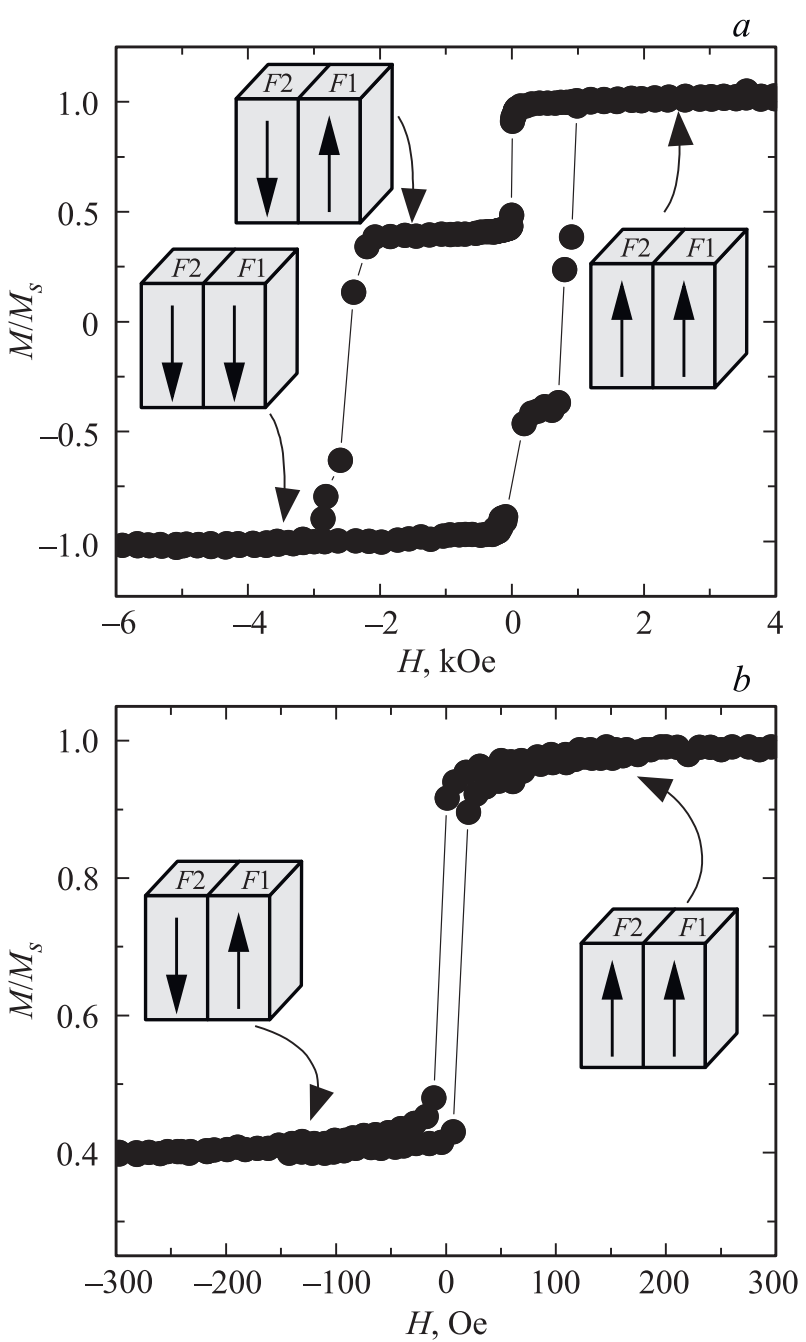

Рис. 6. (а) Магнитная петля гистерезиса для образца 3b, измеренная после процедуры охлаждения от комнатной температуры до $T=10 \mathrm{~K}$ в магнитном поле $+4 \mathrm{kOe}$. $(b)$ Малая петля гистерезиса для того же образца, связанная с изменением намагниченности свободного слоя Ру2 в магнитном поле от $+0.3 \mathrm{kOe}$ до $-0.3 \mathrm{kOе}$ и обратно. 

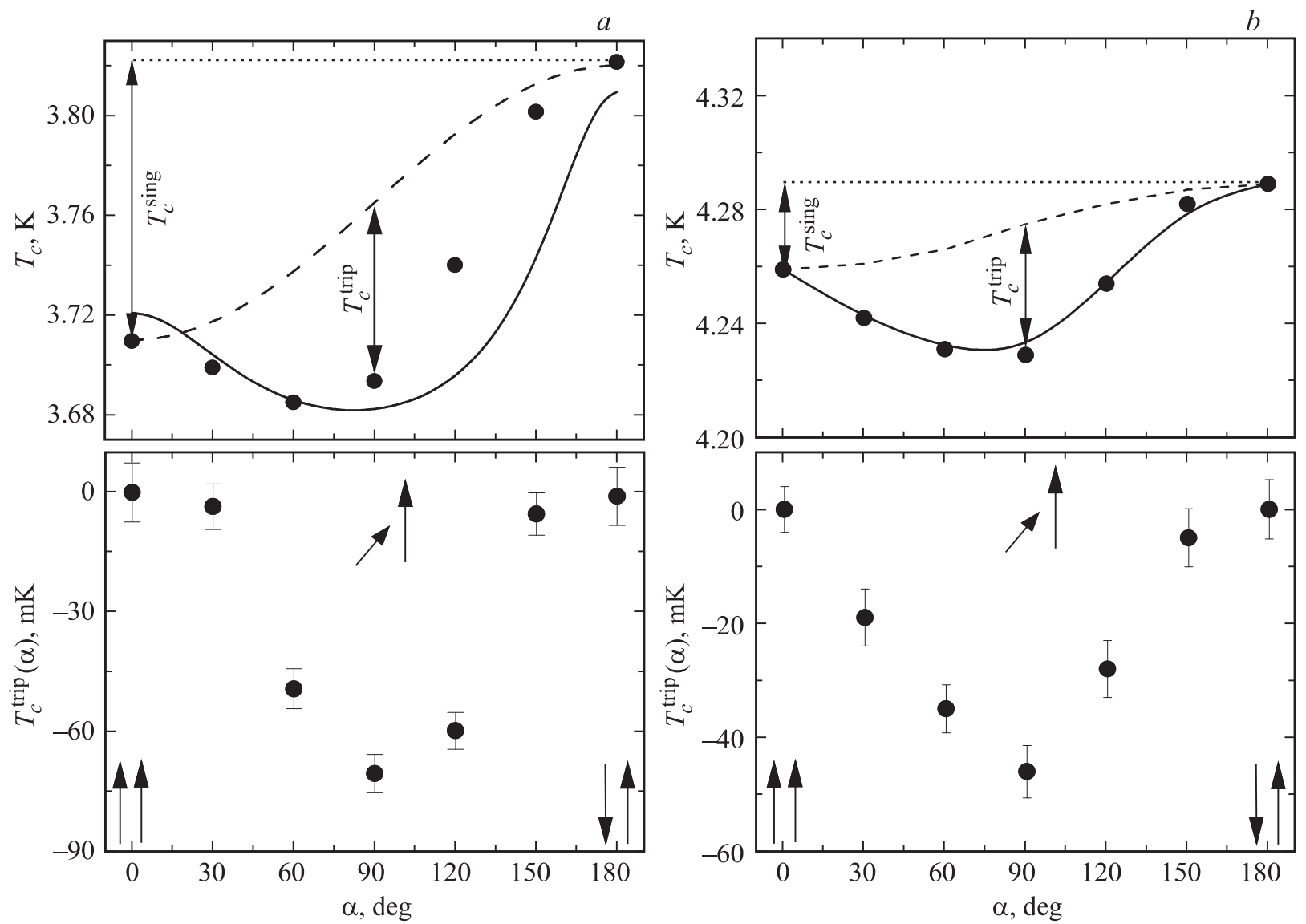

Рис. 7. $(a)$ и $(b)$ температурные зависимости сверхпроводящих переходов от угла между намагниченностями ферромагнитных слоев для образцов $3 \mathrm{a}$ и $3 \mathrm{~d}$ соответственно. Верхние графики: угловые зависимости $T_{c}$, измеренные во внешнем магнитном поле $H_{0}=+100$ Ое (кружки); реперные кривые показаны штриховой линией (см. уравнение (1)); теоретические кривые, рассчитанные согласно теории Фоминова и др. (см. [19]) показаны сплошной линией. Нижние графики: угловые зависимости $\Delta T_{c}^{\text {trip }}-$ разницы между фактическим значением $T_{c}$ и реперной кривой для образцов.

процедуры охлаждения в магнитном поле $+4 \mathrm{kOe}$ магнитное поле изменялось от +0.3 до $-0.3 \mathrm{kOе}$ и обратно до значения $+0.3 \mathrm{kOe}$. При этом происходило измерение намагниченности. Таким образом, в этом диапазоне полей все изменения суммарной намагниченности образца обусловлены слоем Ру2 с незафиксированной намагниченностью. Из этих данных следует, что после процедуры охлаждения образца в поле параллельная и антипараллельная ориентации могут быть гарантировано достигнуты путем приложения магнитного поля +0.1 и $-0.1 \mathrm{kOе} \mathrm{соответственно.}$

Далее исследовались транспортные свойства третьей серии образцов. Была исследована зависимость $T_{c}$ от угла $\alpha$ между намагниченностями ферромагнитных слоев Ру1 и Ру2 в поле $H_{0}=+100$ Ое, приложенном в плоскости образца. Наиболее интересные результаты по зависимости $T_{c}(\alpha)$ удалось получить для образцов $3 \mathrm{a} \mathrm{CoO}_{x} / \mathrm{Py}(3) / \mathrm{Cu}(4) / \mathrm{Py}(0.6) / \mathrm{Cu}(2) / \mathrm{Pb}(70)$ и $3 \mathrm{~d}$ $\mathrm{CoO}_{x} / \mathrm{Py}(3) / \mathrm{Cu}(4) / \mathrm{Py}(3) / \mathrm{Cu}(2) / \mathrm{Pb}(70)$ (см. рис. 7).

Как видно из рис. 7 , угловые зависимости $T_{c}$ этих образцов имеют немонотонный характер. Зависимость $T_{c}(\alpha)$ проходит через минимум в промежутке между $\mathrm{P}\left(\alpha=0^{\circ}\right)$ и $\mathrm{AP}\left(\alpha=180^{\circ}\right)$ вблизи ортогональной конфигурации намагниченностей. В образце $3 \mathrm{a}$ величина эффекта спинового клапана $\Delta T_{c}$ при изменении взаимной ориентации намагниченностей от АР к ортогональной ориентации превышает ширину сверхпроводящего перехода. В связи с этим в такой структуре возникает возможность полного включения и выключения сверхпроводящего тока, как показано на рис. 8, $a$. Полное включение/выключение сверхпроводящего тока, благодаря сочетанию стандартного и триплетного эффекта спинового клапана, продемонстрировано на pис. $8, b$. Температурная зависимость $\Delta R=R\left(\alpha=90^{\circ}\right)$ $-R\left(\alpha=180^{\circ}\right)$ показывает, что в перпендикулярной конфигурации намагниченностей в температурном интервале 3.6-3.66 K наблюдается полное переключение по отношению к антипараллельной конфигурации.

3.3. „Изолированный“ триплетный вклад в эффект сверхпроводящего спинового клапана. В исследованных образцах ДТК дает вклад в эффект спинового клапана. Однако триплетный вклад сосуществует со стандартным. Как было показано ранее, амплитуда стандартного эффекта спинового клапана может быть подавлена до нуля при опреде- 


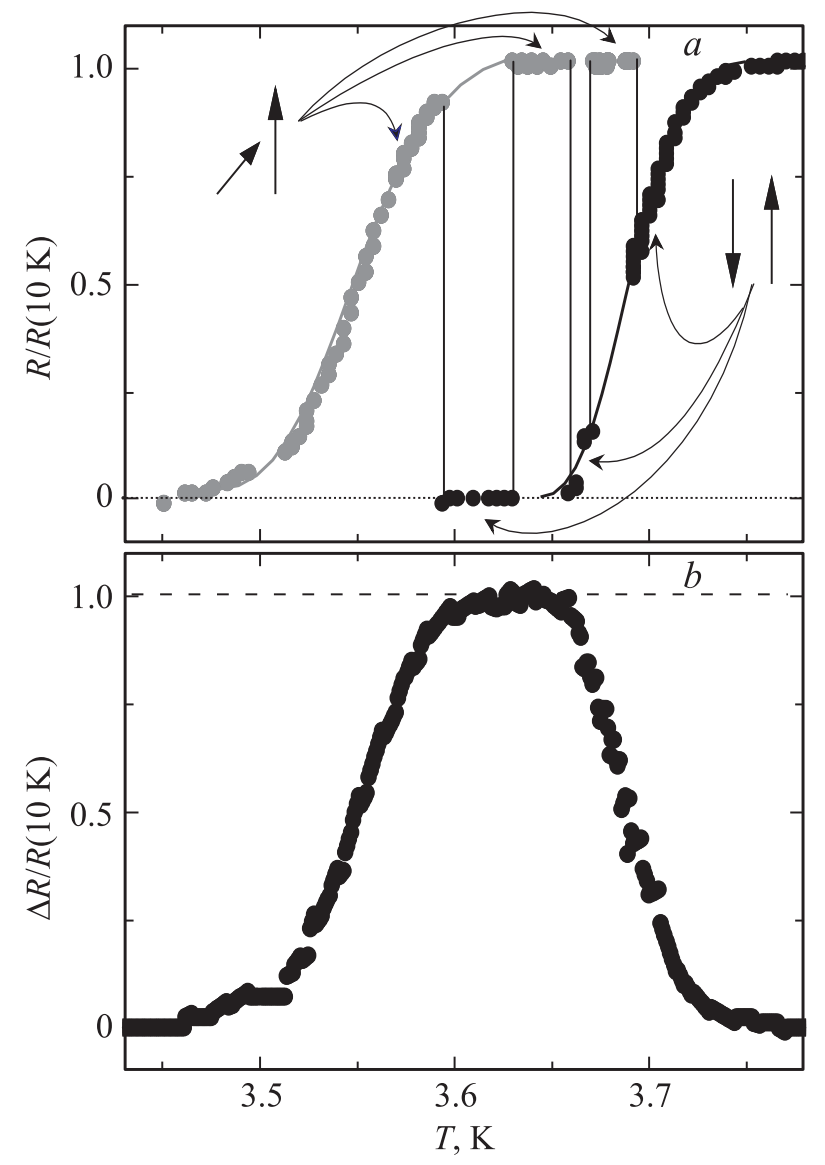

Рис. 8. (a) Переключение между нормальным и сверхпроводящим состояниями для образца За путем медленного понижения температуры в пределах $\Delta T_{c}$ и резкого изменения направления магнитного поля $\alpha$ между $180^{\circ}$ (темные кружки) и $90^{\circ}$ (светлые кружки). (b) Температурная зависимость $\Delta R=R\left(\alpha=90^{\circ}\right)-R\left(\alpha=180^{\circ}\right)$, демонстрирующая полное переключение сверхпроводящего тока в температурном интервале $3.6-3.66 \mathrm{~K}$.

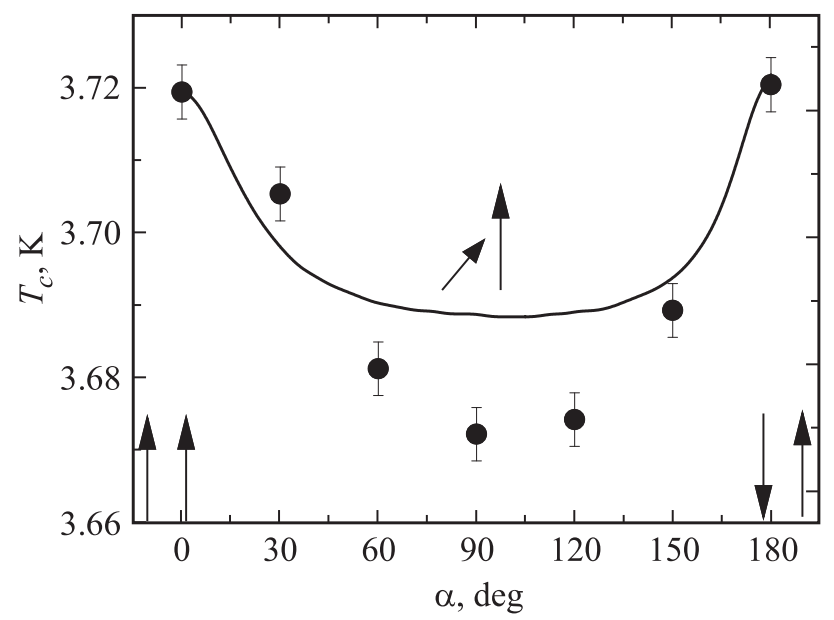

Рис. 9. Образец $3 \mathrm{c} \quad \mathrm{CoO}_{x} / \mathrm{Py}(3) / \mathrm{Cu}(4) / \mathrm{Py}(3) / \mathrm{Cu}(2) / \mathrm{Pb}(70)$ с нулевым стандартным эффектом спинового клапана $\left(T_{c}^{\mathrm{P}}=T_{c}^{\mathrm{AP}}\right)$. Угловая зависимость $T_{c}(\alpha)$, вызванная дальнодействующей триплетной компонентой сверхпроводящего конденсата, показана кружками. Сплошная линия - теоретическая кривая. ленной толщине второго ферромагнетика, вследствие осцилляционного поведения $T_{c}\left(d_{\mathrm{F} 2}\right)$ [26,27]. Эти осцилляции вызваны интерференцией парной волновой функции, на границе раздела F2/S c падающей на нее и отраженной от границы F1/A2. Для структуры $\mathrm{CoO}_{x} / \mathrm{Fe} 1 / \mathrm{Cu} / \mathrm{Fe} 2 / \mathrm{Cu} / \mathrm{Pb}$ нулевое значение $\Delta T_{c}$ достигается в интервале $d_{\mathrm{Fe} 2} \approx 0.8-1 \mathrm{~nm}[22,23]$. Из анализа данных по $T_{c}\left(d_{\text {Ру }}\right)$ был сделан вывод о том, что глубина проникновения куперовских пар в слой пермаллоя превышает величину, полученную для железа в 2 раза. Это означает, что $\Delta T_{c}=0$ для образцов с пермаллоем должно достигаться при $d_{\text {Ру2 }} \approx 1.5-1.7 \mathrm{~nm}$. И в самом деле, для образца $3 \mathrm{c} \mathrm{CoO}_{x} / \mathrm{Py}(3) / \mathrm{Cu}(4) / \mathrm{Py}(1.7) / \mathrm{Cu}(2) / \mathrm{Pb}(70)$ удалось наблюдать изолированный триплетный вклад в эффект спинового клапана (см. рис. 9). Угловая зависимость $T_{c}(\alpha)$ для этого образца представляла собой кривую, у которой $T_{c}$ при $\alpha=0^{\circ}$ и $\alpha=180^{\circ}$ совпадает (стандартный эффект спинового клапана $\left.\Delta T_{c}=T_{c}^{\mathrm{AP}}-T_{c}^{\mathrm{P}}=0\right)$. В то же время при неколлинеарной конфигурации намагниченностей слоев Ру1 и Ру2 зависимость $T_{c}(\alpha)$ имеет минимум. Сравнение этих данных с теорией при использовании тех же параметров, что и для описания данных, полученных для образцов на рис. 7, показало удовлетворительное согласие (см. сплошная линия на рис. 9). Разница была лишь в $d_{\text {Ру2, }}$ которая была взята равной $1.7 \mathrm{~nm}$. Таким образом, была продемонстрирована возможность изоляции (отдельного наблюдения) триплетного вклада в эффект спинового клапана. Создание гетероструктуры S/F, в которой интерференция парных волновых функций делает вклад синглетной компоненты в эффект спинового клапана неэффективным с точки зрения влияния на $T_{c}$, является интересной возможностью.

\section{4. Обсуждение экспериментальных результатов}

4.1. Обсуждение данных по исследованию влияния морфологии сверхпроводящего слоя на свойства образцов. На основании вышеизложенного можно сделать вывод, что структуры сверхпроводящего спинового клапана $\mathrm{CoO}_{x} / \mathrm{Py} 1 / \mathrm{Cu} / \mathrm{Py} 2 /$ $\mathrm{Cu} / \mathrm{Pb}$, приготовленные при температуре подложки $150 \mathrm{~K}$, являются наиболее перспективными образцами для наблюдения максимальных значений величины эффекта сверхпроводящего спинового клапана и триплетной сверхпроводимости. В связи с этим, в дальнейшем изучались именно такие конструкции сверхпроводящего спинового клапана.

Результаты на рис. 5, $a, b$ демонстрируют заметную роль морфологии сверхпроводящего слоя на величину эффекта сверхпроводящего спинового клапана $\Delta T_{c}$. Очевидно, что это не связано с различной степенью подавления $T_{c}$ в образцах с шероховатым и гладким сверхпроводящим слоем, так как зависимость $T_{c}\left(d_{S}\right)$ на рис. 4 оказалась нечувствительной к морфологии сверхпроводящего слоя. Другой причиной может служить 
различная площадь контакта между сверхпроводником и ферромагнетиком на границе $\mathrm{S} / \mathrm{F}$ в случае шероховатого и гладкого S-слоя. Однако, согласно теоретическим моделям (см. $[18,28])$, площадь контакта не участвует в физических процессах, имеющих отношение к эффекту сверхпроводящего спинового клапана. Вероятнее всего, морфология S-слоя влияет на осцилляционное поведение парной волновой функции сверхпроводящего конденсата в ферромагнетике, которое присуще эффекту близости S/F. Сверхпроводящий конденсат в трехслойных системах F1/F2/S описывается аномальной функцией Грина в S- и F-слоях. Куперовская пара, проникая в F-слой из S-слоя, приобретает конечный импульс, который создает пространственную осцилляцию функции Грина в F-слое. Парная волновая функция, идущая через границу раздела $\mathrm{F} 2 / \mathrm{S}$, интерферирует с функцией с отраженной от границы F1/F2. Величина эффекта сверхпроводящего спинового клапана напрямую связана с интерференционной картиной, возникающей в данном процессе (см. [26,27]). Неоднородность в толщине сверхпроводящего слоя, состоящего из перекрывающихся островков размеров $0.2-1 \mu \mathrm{m}$ (см. рис. 1 ), может быть больше, чем длина свободного пробега электронов и длина когерентности $\xi_{S}$. В таком случае, естественно, что различного рода структурные неоднородности могут создать неоднородность аномальной функции Грина в плоскости S-слоя. Поскольку функции Грина в F- и $\mathrm{S}$-слоях связаны через граничные условия S/F, то неоднородность парной волновой функции в S-слое передастся в F-слой. Неоднородность аномальной функции Грина в F-слое приведет к нарушению стабильной интерференционной картины в F-слое, что, в свою очередь, приведет к подавлению эффекта сверхпроводящего спинового клапана. Таким образом, результаты исследований демонстрируют важную роль морфологии сверхпроводящего слоя на величину эффекта сверхпроводящего спинового клапана в структурах F1/F2/S.

4.2. Обсуждение данных по эффекту спинового клапана. Из рис. 7 видно, что величина стандартного эффекта сверхпроводящего спинового клапана для образца $3 \mathrm{a} \Delta T_{c}=110 \mu \mathrm{m}$, а для образца $3 \mathrm{~d}$ $\Delta T_{c}=30 \mu \mathrm{mK}$. Такая разница в величине $\Delta T_{c}$ связана с тем, что при толщине $d_{\text {Ру2 }}=3 \mathrm{~nm}$ в образце $3 \mathrm{~d}$ волновая функция куперовских пар сверхпроводника почти полностью затухает в слое ферромагнетика Ру2, а значит слой Ру1 оказывает слабое влияние на сверхпроводимость и, как следствие, на величину эффекта сверхпроводящего спинового клапана $\Delta T_{c}$. Это согласуется с тем, что в соответствии с теорией, с уменьшением толщины $d_{\text {Ру2 }}$ величина эффекта $\Delta T_{c}$ возрастает.

Как можно заметить из рис. 7, при изменении взаимной ориентации намагниченностей от параллельной $\mathrm{P}$ $\left(\alpha=0^{\circ}\right)$ к $\mathrm{AP}\left(\alpha=180^{\circ}\right) T_{c}$ изменяется немонотонно, проходя через минимум при неколлинеарной ориентации. Согласно теории (см. [19]), характерный минимум в зависимости $T_{c}(\alpha)$, который наиболее ярко проявляется вблизи $\alpha=90^{\circ}$, однозначно свидетельствует о генерации дальнодействующей триплетной компоненты
(ДТК) в сверхпроводящем конденсате. Если предположить, что триплетной компоненты нет (хотя, согласно теории, их возникновение неизбежно), можно ожидать, что зависимость $T_{c}(\alpha)$ будет монотонной. Исходя из общих соображений, $T_{c}$ должна быть функцией $\alpha^{2}$ и $(\pi-\alpha)^{2}$ при изменении угла от 0 до $\pi$. Таким образом, зависимость $T_{c}(\alpha)$ можно выразить через $T_{c}^{\mathrm{P}}$ и $T_{c}^{\mathrm{AP}}$ следующим образом:

$$
T_{c}^{(\mathrm{ref})}(\alpha)=T_{c}^{\mathrm{P}} \cos ^{2}(\alpha / 2)+T_{c}^{\mathrm{AP}} \sin ^{2}(\alpha / 2) .
$$

Эта воображаемая кривая представлена штриховой линией на рис. 7 на зависимостях $T_{c}(\alpha)$. Назовем эту кривую реперной. Отклонение фактического значения $T_{c}$ от этой реперной кривой показано на рис. 7 на зависимостях $\Delta T_{c}^{\text {trip }}(\alpha)$. Величина этого отклонения демонстрирует вклад ДТК в величину эффекта спинового клапана. Обозначим ее как $\Delta T_{c}^{\text {trip }}$ и получаем, что различие в $T_{c}$ между антипараллельной и перпендикулярной ориентациями составляет уже $130 \mathrm{mK}$ для образца $3 \mathrm{a}$ и $60 \mathrm{mK}$ для образца $3 \mathrm{~d}$. Это означает, что ДТК дает заметный вклад в эффект спинового клапана.

Во всех предыдущих работах нашей группы (см. $[19,22,23,26,27])$ экспериментальные данные по $T_{c}$ сравнивались с параметром теории $W$, который определяет степень подавления сверхпроводимости ферромагнитным слоем (см. [16]). Эффективное граничное условие для реальной компоненты аномальной функции Грина может быть записано как

$$
\xi_{S} \frac{d f_{0}}{d x}=W f_{0} .
$$

Это рассмотрение позволило продемонстрировать качественное согласие теории (параметра $W$ ) и эксперимента $\left(T_{c}\right)$ без вычисления самой критической температуры. Здесь же, впервые, проведен прямой сравнительный анализ теоретически рассчитанного и экспериментально измеренного значения $T_{c}$. Для теоретического расчета $T_{c}(\alpha)$ была использована теория Фоминова и др. (см. [18]) на случай произвольной прозрачности границы F2/S (см. [22,23]). Данный расчет представлен сплошной линией на рис. 7. Для теоретического расчета были использованы следующие параметры: длины когерентности для S- и F-слоев $\xi_{S}=41 \mathrm{~nm}, \xi_{\mathrm{F}}=13 \mathrm{~nm}$; толщина сверхпроводящего слоя $d_{\mathrm{Pb}}=73.5 \mathrm{~nm}$; температура перехода в сверхпроводящее состояние массивного свинца $T_{c}^{\mathrm{Pb}}=7.2 \mathrm{~K}$; параметры прозрачности границы $\mathrm{Py} 2 / \mathrm{Pb} \gamma=0.734$ и $\gamma_{b}=1.8$; величина обменного поля в ферромагнетике $h=0.3 \mathrm{eV}$. При теоретических расчетах было обнаружено, что теория требует толщину

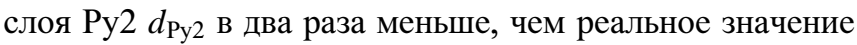
толщины слоя. Данное обстоятельство, скорее всего, обусловлено тем, что слой Ру2 окружен с обеих сторон слоями меди, и, вследствие взаимной диффузии атомов меди и пермаллоя, эффективная толщина слоя Ру2 оказывается уменьшенной. Как видно из рис. 7 и 9 теория воспроизводит основные черты экспериментальных зависимостей. Заметное расхождение теории и экс- 
перимента может быть обусловлено двумя причинами. В теории Фоминова и др. [18] в качестве F-слоев рассматривался слабый ферромагнетик, а пермаллой не является таковым. Кроме того, мы не учитывали ограничение прозрачности границы раздела Рy1/Cu/Py2, как это делалось в теоретических работах $[28,29]$, поскольку для нас этот параметр явился бы свободным. В отличие от параметров, характеризующих прозрачность границы раздела Ру $2 / \mathrm{Pb}$, мы не можем оценить эту величину из эксперимента.

\section{5. Заключение}

Итак, в ходе исследований, представленных в настоящей работе, были получены следующие основные результаты.

Во-первых, было установлено существенное влияние морфологии сверхпроводящего слоя на величину эффекта сверхпроводящего спинового клапана. Исследования показывают, что использование гладкого сверхпроводящего слоя вместо шероховатого позволяет увеличить величину $\Delta T_{c}$ с 10 до $100 \mathrm{mK}$.

Во-вторых, была экспериментально исследована дальнодействующая триплетная компонента в образцах сверхпроводящего спинового клапана $\mathrm{CoO}_{x} / \mathrm{Py} 1 / \mathrm{Cu} /$ $\mathrm{Py} 2 / \mathrm{Cu} / \mathrm{Pb}$. Было получено полное переключение между сверхпроводящим и нормальным состояниями. Переключение было вызвано сочетанием стандартного и триплетного эффектов спинового клапана при изменении взаимной ориентации намагниченностей слоев Ру1 и Ру2 от антипараллельной к перпендикулярной ориентации.

В-третьих, впервые проведено прямое сравнение теоретически рассчитанного и экспериментального полученного значения $T_{c}$.

\section{Список литературы}

[1] S. Oh, D. Youm, M.R. Beasley. Appl. Phys. Lett. 71, 2376 (1997).

[2] M. Eschrig. Phys. Today 64, 43 (2011).

[3] M.G. Blamire, J.W.A. Robinson. J. Phys.: Condens. Matter 26, 453201 (2014).

[4] J. Linder, J.W.A. Robinson. Nature Phys. 11, 307 (2015).

[5] V.I. Zdravkov, J. Kehrle, G. Obermeier, D. Lenk, H.-A. Krug von Nidda, C. Müller, M.Yu. Kupriyanov, A.S. Sidorenko, S. Horn, R. Tidecks, L.R. Tagirov. Phys. Rev. B 87, 144507 (2013).

[6] A.A. Jara, C. Safranski, I.N. Krivorotov, C.-T. Wu, A.N. MalmiKakkada, O.T. Valls, K. Halterman. Phys. Rev. B 89, 184502 (2014).

[7] X.L. Wang, A.Di Bernardo, N. Banerjee, A. Wells, F.S. Bergeret, M.G. Blamire, J.W.A. Robinson. Phys. Rev. B 89, 140508(R) (2014).

[8] P.V. Leksin, N.N. Garif'yanov, I.A. Garifullin, J. Schumann, H. Vinzelberg, V. Kataev, R. Klingeler, O.G. Schmidt, B. Büchner. Appl. Phys. Lett. 97, 102505 (2010).

[9] Bin Li, N. Roschewsky, B.A. Assaf, Marius Eich, M. EpsteinMartin, D. Heiman, M. Munzenberg, J. Moodera. Phys. Rev. Lett. 110, 097001 (2013).
[10] A. Singh, S. Voltan, K. Lahabi, J. Aarts. Phys. Rev. X 5, 021019 (2015).

[11] Y. Gu, J.W.A. Robinson, M. Bianchetti, N.A. Stelmashenko, D. Astill, F.M. Grosche, J.L. MacManus-Discoll, M.G. Blamire. APL Matter 2, 046103 (2014).

[12] Y. Gu, G.B. Halász, J.W.A. Robinson, M.G. Blamire. Phys. Rev. Lett. 115, 067201 (2015).

[13] S. Mironov, A. Buzdin. Phys. Rev. B 92, 184506 (2015).

[14] F.S. Bergeret, A.F. Volkov, K.B. Efetov. Rev. Mod. Phys. 77, 1321 (2005).

[15] A.I. Buzdin. Rev. Mod. Phys. 77, 935 (2005).

[16] K.B. Efetov, I.A. Garifullin, A.F. Volkov, K. Westerholt. Proximity Effects in Ferromagnet / Superconductor Heterostructures: Magnetic Heterostructures. Advances and Perspectives in Spinstructures and Spintransport. Series Springer Tracts in Modern Physics. Springer, Berlin (2007). V. 227. P. 251-289.

[17] K.B. Efetov, I.A. Garifullin, A.F. Volkov, K. Westerholt. SpinPolarized Electrons in the superconductor/ferromagnet hybrid structures: Magnetic Nanostructures. Spin Dynamic and Spin Transport. Series Springer Tracts in Modern Physics. / Eds H. Zabel, M. Farle. Springer-Verlag, Berlin Heidelberg (2013). V. 246. P. 85-118

[18] Ya.V. Fominov, A.A. Golubov, T.Yu. Karminskaya, M.Yu. Kupriyanov, R.G. Deminov, L.R. Tagirov. Письма в ЖЭТФ 91, 329 (2010).

[19] P.V. Leksin, N.N. Garif'yanov, I.A. Garifullin, Ya.V. Fominov, J. Schumann, Y. Krupskaya, V. Kataev, O.G. Schmidt, B. Büchner. Phys. Rev. Lett. 109, 057005 (2012).

[20] M.G. Flokstra, T.C. Cunningham, J. Kim, N. Satchell, G. Burnell, P.J. Curran, S.J. Bending, C.J. Kinane, J.F.K. Cooper, S. Langridge, A. Isidori, N. Pugach, M. Eschrig, S.L. Lee. Phys. Rev. B 91, 060501 (2015).

[21] N. Banerjee, C.B. Smiet, R.G.J. Smits, A. Ozaeta, F.S. Bergeret, M.G. Blamire, J.W.A. Robinson. Natur. Commun. 5, 3048 (2014).

[22] P.V. Leksin, A.A. Kamashev, N.N. Garif'yanov, I.A. Garifullin, Ya.V. Fominov, J. Schumann, C. Hess, V. Kataev, B. Büchner. Письма в ЖЭТФ 97, 549 (2013).

[23] P.V. Leksin, N.N. Garif'yanov, A.A. Kamashev, Ya.V. Fominov, J. Schumann, C. Hess, V. Kataev, B.Büchner, I.A. Garifullin. Phys. Rev. B 91, 214508 (2015).

[24] P.V. Leksin, A.A. Kamashev, J. Schumann, V. Kataev. J. Thomas, B. Büchner, I.A. Garifullin. Nano Res. (2016) (accepted); (arXiv:1510.04846)

[25] Y.V. Fominov, N.M. Chtchelkatchev, A.A. Golubov. Phys. Rev. B 66, 014507 (2002).

[26] P.V. Leksin, N.N. Garifyanov, I.A. Garifullin, J. Schumann, V. Kataev, O.G. Schmidt, B. Büchner. Phys. Rev. Lett. 106, 067005 (2011).

[27] P.V. Leksin, N.N. Garifyanov, I.A. Garifullin, J. Schumann, V. Kataev, O.G. Schmidt, B. Büchner. Phys. Rev. B 85, 024502 (2012).

[28] R.G. Deminov, L.R. Tagirov, R.R. Gaifullin, T.Yu. Karminskaya, M.Yu. Kupriyanov, Ya.V. Fominov, A.A. Golubov. J. Magn. Magn. Mater. 373, 16 (2015).

[29] G. Deminov, L.R. Tagirov, R.R. Gaifullin, Ya.V. Fominov, T.Yu. Karminskaya, M.Yu. Kupriyanov, A.A. Golubov. Solid State Phenomena 233-234, 745-749 (2015). 